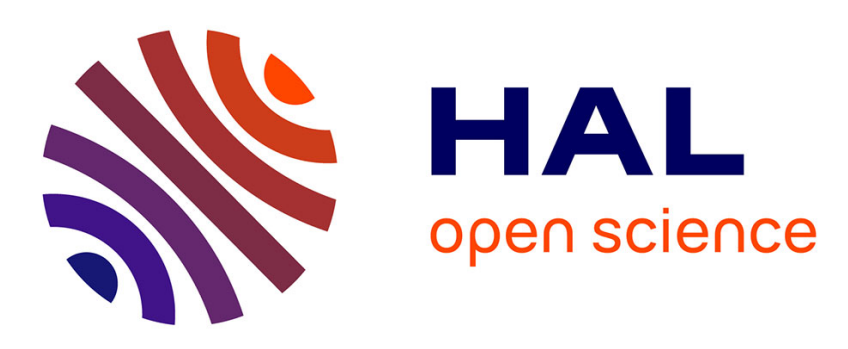

\title{
Vortex solutions of the defocusing discrete nonlinear Schrödinger equation
}

\author{
Jesus Cuevas, Guillaume James, Panayotis G. Kevrekidis, Kody Law
}

\section{To cite this version:}

Jesus Cuevas, Guillaume James, Panayotis G. Kevrekidis, Kody Law. Vortex solutions of the defocusing discrete nonlinear Schrödinger equation. ICNAAM 2009 - 7th International Conference on Numerical Analysis and Applied Mathematics, Sep 2009, Rethymno, Crete, Greece. pp.135-138, 10.1063/1.3241338 . hal-00769565

\section{HAL Id: hal-00769565 https://hal.science/hal-00769565}

Submitted on 2 Sep 2019

HAL is a multi-disciplinary open access archive for the deposit and dissemination of scientific research documents, whether they are published or not. The documents may come from teaching and research institutions in France or abroad, or from public or private research centers.
L'archive ouverte pluridisciplinaire HAL, est destinée au dépôt et à la diffusion de documents scientifiques de niveau recherche, publiés ou non, émanant des établissements d'enseignement et de recherche français ou étrangers, des laboratoires publics ou privés. 


\title{
Vortex Solutions of the Defocusing Discrete Nonlinear Schrödinger Equation
}

\author{
J. Cuevas*, G. James ${ }^{\dagger}$, P.G. Kevrekidis ${ }^{* *}$ and K.J.H. Law** \\ ${ }^{*}$ Grupo de Física No Lineal. Departamento de Física Aplicada I. Escuela Universitaria Politécnica. \\ Universidad de Sevilla. C/Virgen de África, 7. 41011 Sevilla, Spain \\ ${ }^{\dagger}$ Institut National Polytechnique de Grenoble and CNRS, Laboratoire Jean Kuntzmann (UMR 5224), tour IRMA, \\ BP 53, 38041 Grenoble Cedex 9, France \\ ** Department of Mathematics and Statistics, University of Massachusetts, Amherst MA 01003-4515, USA
}

\begin{abstract}
We consider the existence, stability and dynamical evolution of dark vortex states in the two-dimensional defocusing DNLS equation, a model of interest both to atomic physics and to nonlinear optics. Our considerations are chiefly based on initializing such vortex configurations at the anti-continuum limit of zero coupling between adjacent sites, and continuing them to finite values of the coupling. Discrete defocusing vortices become unstable past a critical coupling strength and, subsequently feature a cascade of alternating stabilization-destabilization windows for any finite lattice.
\end{abstract}

Keywords: DNLS equation, Vortices, Existence, Stability

PACS: $05.45 . \mathrm{Yv}, 42.65 . \mathrm{Tg}, 03.75 . \mathrm{Lm}$

\section{INTRODUCTION}

The study of vortices and their existence, stability and dynamical properties has been a central theme of study in the area of Bose-Einstein condensates (BECs) [1]. In particular, the remarkable experiments illustrating the generation of vortices [2] and of very robust lattices thereof [3] have stirred a tremendous amount of activity in this area in the past few years, that has by now been summarized in various reviews and books [4,5]. Much of this activity has been centered around the robustness of vortex structures in the context of the mean-field dynamics of the BECs (which are controllably accurately described by a nonlinear Schrödinger (NLS) equation) in the presence of many of the potentials that are relevant to the trapping of atomic BECs including parabolic traps [1] and periodic optical lattice ones [6].

On the other hand, the BECs in the presence of periodic potentials have been argued to be well-approximated by models of the discrete nonlinear Schrödinger (DNLS) type (i.e., resembling the finite-difference discretization of the continuum equation) $[7,8]$. In that regard, to understand the existence and stability properties of vortices in the presence of periodic potentials, it would be interesting to analyze the discrete analog of the relevant NLS equation. This is also interesting from a different perspective in this BEC context, namely that if finite-difference schemes are employed to analyze the properties of the continuum equation, it is useful to be aware of features introduced by virtue of the discretization.

However, it should be stressed that this is not a problem of restricted importance in the context of quantum fluids; it is also of particular interest in nonlinear optics where two-dimensional optical waveguide arrays have been recently systematically constructed e.g. in fused silica in the form of square lattices [9], whereby discrete solitons can be excited. By analogy to their one-dimensional counterparts of discrete dark solitons, which have been created in defocusing waveguide arrays with the photovoltaic nonlinearity [10], we expect that it should be possible to excite discrete dark vortices in defocusing two-dimensional waveguide arrays.

An especially interesting feature of dark solitons that was observed initially in [11] is that on-site discrete dark solitons are stable for sufficiently coarse lattices, but they become destabilized beyond a certain coupling strength among adjacent lattice sites and remain so until the continuum limit where they are again restabilized (as the point spectrum eigenvalue that contributes to the instability becomes zero due to the restoration of the translational invariance in the continuum problem) $[11,12]$. It is therefore of interest to examine if the instability mechanisms of discrete defocusing vortices are of this same type or are potentially different and how the relevant stability picture is modified as a function of the inter-site coupling strength.

It is this problem of the existence, stability and continuation of the vortex structures as a function of coupling strength that we examine in the present work. We consider, in particular, a two-dimensional discrete nonlinear Schrödinger 
equation

$$
i \frac{d \psi_{n, m}}{d t}-\left|\psi_{n, m}\right|^{2} \psi_{n, m}+\varepsilon \Delta \psi_{n, m}=0
$$

where $\Delta \psi_{n, m}=\psi_{n+1, m}+\psi_{n-1, m}+\psi_{n, m+1}+\psi_{n, m-1}-4 \psi_{n, m}$ is the discrete Laplacian and $\varepsilon$ is a coupling constant. We study the defocusing case when $\varepsilon>0$ and look for time-periodic solutions with frequency $\omega$. Using the ansatz $\psi_{n, m}(t)=\sqrt{\omega} \phi_{n, m} e^{-i \omega t}$, we obtain

$$
C \Delta \phi_{n, m}+\left(1-\left|\phi_{n, m}\right|^{2}\right) \phi_{n, m}=0
$$

where we have set $C=\varepsilon / \omega$. The coupling parameter $C>0$ determines the strength of discreteness effects. The limit $C \rightarrow+\infty$ corresponds to the continuum (stationary) Gross-Pitaevskii equation and the case $C \rightarrow 0$ corresponds to the so-called anti-continuum (AC) limit [13].

When Eq. (2) is considered on an infinite lattice $\mathbb{Z}^{2}$, we look for solutions satisfying $\left|\phi_{n, m}\right| \rightarrow 1$ when $(n, m) \rightarrow \infty$, for which $\phi_{n, m}$ vanishes at one lattice site, e.g. at $(n, m)=(0,0)$. Such solutions are denoted as discrete vortices, or "dark" vortex solitons. If one trigonometric turn on any path $\operatorname{Max}(|n|,|m|)=\rho$ around the vortex center changes the argument of $\phi_{n, m}$ by $2 \pi S(S \in \mathbb{Z})$, then the vortex is said to have a topological charge (or vorticity) equal to $S$.

In this presentation we numerically investigate the existence and stability of such solutions on a finite lattice of size $N \times N, N$ being large; our analysis is performed as a function of the lattice coupling parameter $C$ and we illustrate how to perform relevant continuations from the AC limit. We mainly focus on numerically computing vortex solutions with vorticity $S=1$ and $S=2$.

\section{NUMERICAL METHOD}

We compute vortex solutions of (2) using the Newton method and a continuation with respect to $C$. The path-following can be initiated either near the continuum limit (for $C$ large) or at the anti-continuum limit $C=0$, since in both cases one is able to construct a suitable initial guess for the Newton method. For relatively high $C$, a suitable initial condition for a vortex with topological charge $S$ is obtained with a Padé approximation developed for the continuum limit in [14]. Once a vortex is found for a given $C$, the solution can be continued by increasing or decreasing $C$. Although this method was found to be efficient, it remains limited to single vortex solutions having explicit continuum approximations. Moreover, when the Newton method is applied to continue these solutions near $C=0$, the Jacobian matrix becomes ill-conditioned (and non-invertible for $C=0$ ) and the iteration does not converge.

In what follows we introduce a different method having a wider applicability, and for which the above mentioned singularity is removed. We consider a finite $N \times N$ lattice with $(n, m) \in \Gamma=\{-M, \ldots, M\}^{2}(N=2 M+1)$, equipped with fixed-end boundary conditions given below. We set $\phi_{n, m}=R_{n, m} e^{i \theta_{n, m}}$, with:

$$
\begin{array}{rll}
R_{n, m}=1 & \text { for } & \operatorname{Max}(|n|,|m|)=M \\
\theta_{n, m}=S \alpha_{n, m} & \text { for } & \operatorname{Max}(|n|,|m|)=M
\end{array}
$$

and

$$
\alpha_{n, m}= \begin{cases}\arctan (m / n)+\frac{3 \pi}{2} & \text { for } n \geq 1, \\ \arctan (m / n)+\frac{\pi}{2} & \text { for } n \leq-1 \\ \frac{\pi}{2}(1-\operatorname{sign}(m)) & \text { for } n=0\end{cases}
$$

For $C=0$, a single vortex at $(n, m)=(0,0)$ corresponds to fixing $R_{0,0}=0$ and $R_{n, m}=1$ everywhere else. As deduced in [15], angles $\theta_{n, m}$ fulfill

$$
\sin \left(\theta_{n+1, m}-\theta_{n, m}\right)-\sin \left(\theta_{n, m}-\theta_{n-1, m}\right)+\sin \left(\theta_{n, m+1}-\theta_{n, m}\right)-\sin \left(\theta_{n, m}-\theta_{n, m-1}\right)=0
$$

with $(n, m) \in \Gamma \backslash\{(0, \pm 1),( \pm 1,0),( \pm M, m),(n, \pm M)\}$, supplemented by the four following relations at $(n, m)=$ $(0, \pm 1),( \pm 1,0)$

$$
\begin{aligned}
& \sin \left(\theta_{1, \pm 1}-\theta_{0, \pm 1}\right)-\sin \left(\theta_{0, \pm 1}-\theta_{-1, \pm 1}\right)+\sin \left(\theta_{0, \pm 2}-\theta_{0, \pm 1}\right)=0 \\
& \sin \left(\theta_{ \pm 2,0}-\theta_{ \pm 1,0}\right)+\sin \left(\theta_{ \pm 1,1}-\theta_{ \pm 1,0}\right)-\sin \left(\theta_{ \pm 1,0}-\theta_{ \pm 1,-1}\right)=0
\end{aligned}
$$



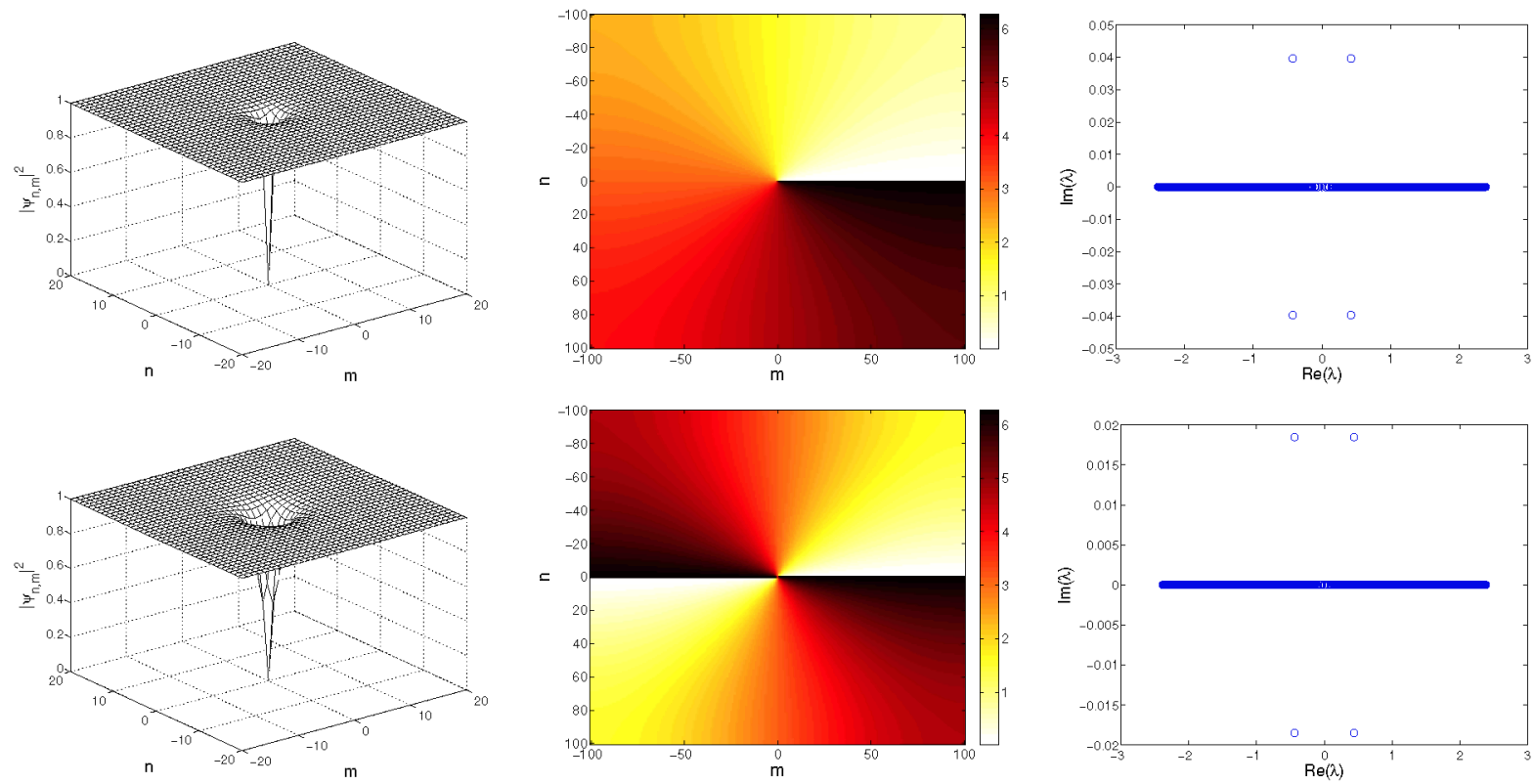

FIGURE 1. Vortex solitons with $C=0.2$ and $S=1$ (top row) and $S=2$ (bottom row). (Left panels) density profile; (center panels) angular dependence; (right panels) spectral plane of stability eigenfrequencies [recall that the presence of eigenfrequencies with non-vanishing imaginary part denotes instability].

Solutions for (5)-(7) are computed by the Newton method, starting from the initial guess $\theta_{n, m}=\alpha_{n, m}$. The initial guess $\phi_{n, m}=R_{n, m} e^{i S \theta_{n, m}}$, can be used to compute a vortex solution of (2) by the Newton method, with Eqs. (3) and (4) acting as boundary conditions. The continuation technique used is a regular parametric continuation, i.e., for each value of $C$ (starting at the anti-continuum limit), we converge to the exact numerical solution, and then we increment the value of $C$ by a small $\delta C$, using the converged solution of the previous step as a good initial guess for the solution of the next one (i.e., of the case with $C+\delta C$ ).

\section{NUMERICAL COMPUTATION OF SINGLE VORTICES}

In this section we analyze the existence and stability of discrete vortices centered on a single site, as a function of the coupling strength $C$ for fixed-end boundary conditions. The stability of the discrete vortex solutions is studied assuming small perturbations in the form of $\delta \psi_{m, n}=\exp (-i t)\left[p_{n, m} \exp (-i \lambda t)+q_{n, m} \exp \left(i \lambda^{*} t\right)\right]$, the onset of instability indicated by the emergence of $\operatorname{Im}(\lambda) \neq 0 ; \lambda$ in this setting denotes the perturbation eigenfrequency. Note that it is sufficient to consider the case $\omega=1$ for stability computations, because this case can always be recovered by rescaling time.

The stability analysis is done fully for the case of configurations of size $41 \times 41$. Subsequently, the procedure is repeated with matrices of size $81 \times 81,121 \times 121,161 \times 161$ and $201 \times 201$. For the latter sizes, it is no longer possible to compute all the eigenvalues at once by a full matrix diagonalization. So, we exploit the sparsity of the system with an Arnoldi iterative diagonalization routine to calculate eigenvalues and corresponding (approximate) eigenvectors.

Figure 1 shows, for $S=1$ and $S=2$ vortices, the profile $\left|\psi_{n, m}\right|^{2}=\left|\phi_{n, m}\right|^{2}=R_{n, m}^{2}$, the angles $\theta_{n, m}$, and the spectral plane of the stability eigenfrequencies. In all cases, $C=0.2$ is shown, which corresponds to unstable solutions. Vortices with $S=1$ and $S=2$ are, respectively, stable for $C<C_{c r} \approx 0.0395$ and $C<C_{c r} \approx 0.0425$. This instability, highlighted in Fig. 2 can be rationalized by analogy with the corresponding stability calculations in the case of dark solitons [11]. At $C=C_{c r}$ the system experiences a Hamiltonian Hopf bifurcation. When $C$ increases, a cascade of Hopf bifurcations takes place due to the interaction of a localized mode with extended modes, as it was observed in one-dimensional dark solitons $[11,16]$. This cascade implies the existence of stability windows between inverse Hopf bifurcations and direct Hopf bifurcations.

For $S=1$ vortices, each one of the bifurcations takes place for decreasing $|\operatorname{Re}(\lambda)|$ when $C$ grows, and, in consequence, the bifurcations cease at a given value of $C$, as $|\operatorname{Re}(\lambda)|$ of the localized mode is smaller than that of the lowest 

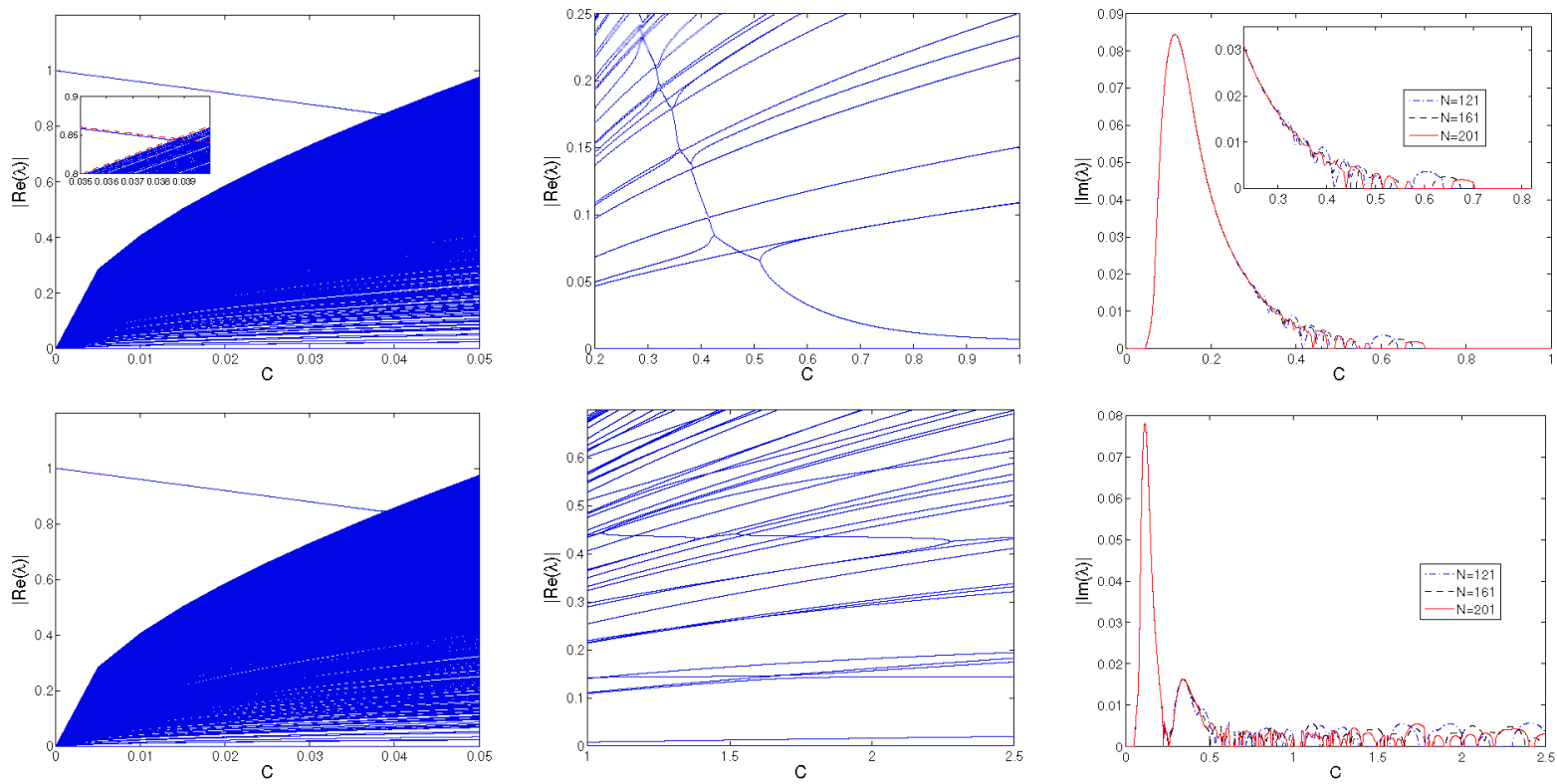

FIGURE 2. Stability for $S=1$ (top row) and $S=2$ (bottom row) vortices. (Left and center panels) Real part of the stability eigenfrequencies. These panels show zooms of two different regions. (Right panels) Largest imaginary part of the stability eigenfrequencies as a function of the coupling strength $C$. This corresponds to the instability growth rate.

extended mode frequency [however, in the infinite domain limit, this eventual restabilization would not take place but for the limit of $C \rightarrow \infty]$. This fact is illustrated in Fig. 2. When the lattice size tends to infinity $(N \rightarrow \infty)$, the linear mode band extends from zero to infinity and becomes dense; thus, we conjecture that these restabilization windows should disappear at this limit. In an effort to numerically suggest this point, we have considered lattices of up to $201 \times 201$ sites for the $S=1$ and $S=2$ vortices and have shown the growth rate of the corresponding instabilities in the right panels of Fig. 2. The maximum growth rate (i.e. the largest imaginary part of the stability eigenfrequencies) takes place at $C \approx 0.115$ for $S=1$ and $S=2$ and being $\operatorname{Im}(\lambda) \approx 0.0845(0.0782)$ for $S=1(S=2)$.

Further insight could be gained by means of a Lyapunov-Schmidt stability analysis [17]. We will consider in the near future this stability study. Another perspective is to consider saturable nonlinearity instead of the cubic one.

\section{REFERENCES}

1. L.P. Pitaevskii and S. Stringari, Bose-Einstein Condensation, Oxford University Press (Oxford, 2003).

2. M. R. Matthews, B. P. Anderson, P. C. Haljan, D. S. Hall, C. E. Wieman, and E. A. Cornell, Phys. Rev. Lett. 83, 2498 (1999).

3. J.R. Abo-Shaeer, C. Raman, W. Ketterle, Phys. Rev. Lett. 88, 070409 (2002).

4. A.L. Fetter and A.A. Svidzinsky, J. Phys. Condens. Matter 13, R135 (2001).

5. P.G. Kevrekidis, D.J. Frantzeskakis and R. Carretero-González (Eds.), Emergent Nonlinear Phenomena in Bose-Einstein Condensates, Springer-Verlag (Heidelberg, 2008).

6. O. Morsch and M. Oberthaler, Rev. Mod. Phys. 78, 179 (2006).

7. F.Kh. Abdullaev, Yu.V. Bludov, S.V. Dmitriev, P.G. Kevrekidis, and V. V. Konotop, Phys. Rev. E 77, 016604 (2008)

8. P.G. Kevrekidis (Ed.), The Discrete Nonlinear Schrödinger Equation: Mathematical Analysis, Numerical Computations and Physical Perspectives. Springer Verlag (Heidelberg, 2009).

9. A. Szameit, J. Burghoff, T. Pertsch, S. Nolte, A Tünnermann, Opt. Express 14, 6055 (2006).

10. E. Smirnov, C.E. Rüter, M. Stepić, D. Kip and V. Shandarov, Phys. Rev. E 74, 065601 (R).

11. M. Johansson and Yu.S. Kivshar. Phys. Rev. Lett. 82, 85 (1999).

12. E.P. Fitrakis, P.G. Kevrekidis, H. Susanto and D.J. Frantzeskakis, Phys. Rev. E 75, 066608 (2007).

13. R.S. MacKay and S. Aubry. Nonlinearity 7, 1623 (1994).

14. N.G. Berloff. J. Phys. A: Math. Gen. 37, 1617 (2004).

15. J. Cuevas, G. James, P.G. Kevrekidis. Physica D 238, 1422 (2009).

16. A. Álvarez, J.F.R. Archilla, J. Cuevas and F.R. Romero. New J. Phys. 4, 72 (2002).

17. D.E. Pelinovsky, P.G. Kevrekidis and D.J. Frantzeskakis. Physica D 212, 20 (2005). 